\title{
Long-term effects of insulin glargine on the risk of breast cancer
}

\author{
S. Suissa • L. Azoulay • S. Dell'Aniello • M. Evans • \\ J. Vora $\cdot$ M. Pollak
}

Received: 6 December 2010 / Accepted: 15 April 2011 /Published online: 26 May 2011

(C) Springer-Verlag 2011

\begin{abstract}
Aims/hypothesis There have been growing concerns regarding the long-term effects of insulin glargine (A21Gly,B31Arg, B32Arg human insulin) on the risk of breast cancer.

Methods We used the UK's General Practice Research Database (GPRD) to identify a cohort of women aged 40 years or over with type 2 diabetes, treated with insulin during 2002-2006 and followed until the first breast cancer diagnosis or 31 December 2009. After the users of insulin glargine had been matched with users of other insulins on age, calendar time and duration of prior insulin use, the HR of breast cancer associated with insulin glargine use was estimated using a Cox proportional hazards model, adjusted for known risk factors for breast cancer.

Results The cohort comprised 15,227 women, including 4,579 glargine users and 10,648 users of other insulins, of
\end{abstract}

S. Suissa $(\bowtie) \cdot$ L. Azoulay $\cdot$ S. Dell'Aniello

Center for Clinical Epidemiology, Lady Davis Research Institute, Jewish General Hospital,

3755 Cote Ste-Catherine,

Montreal, QC, Canada H3T 1E2

e-mail: samy.suissa@mcgill.ca

\section{S. Suissa}

Department of Epidemiology and Biostatistics, McGill University, Montreal, Canada

L. Azoulay $\cdot$ M. Pollak

Department of Oncology, McGill University,

Montreal, QC, Canada

\section{Evans}

University Hospital Llandough,

Cardiff, UK

J. Vora

Royal Liverpool University Hospital,

Liverpool, UK which 246 developed breast cancer during up to 8 years follow-up (incidence rate 4.1 per 1,000 per year). Insulin glargine use was not associated with an increased risk of breast cancer during the first 5 years of use (HR 0.9; $95 \%$ CI $0.7-1.3$ ). The risk tended to increase after 5 years (HR $1.8 ; 95 \%$ CI $0.8-4.0$ ), and significantly so for the women who had been on insulin before starting glargine (HR 2.7; 95\% CI 1.1-6.5).

Conclusions/interpretation The risk of breast cancer in women with type 2 diabetes is not increased during the first 5 years of insulin glargine use. However, longer-term use may increase this risk, particularly in women with longstanding use of insulin before starting insulin glargine.

Keywords Carcinogenicity - Cohort studies - Drug safety · Insulin analogues · Type 2 diabetes

\section{Abbreviations \\ GPRD General Practice Research Database \\ HRT Hormone replacement therapy \\ IGF-I Insulin-like growth factor I}

\section{Introduction}

Insulin analogues, structurally altered human insulins first introduced in the late 1990s to improve control of hyperglycaemia, have been an object of concern regarding their potential carcinogenic effects, particularly those with enhanced affinity for insulin-like growth factor I (IGF-I) receptor and/or altered binding kinetics for the insulin receptor [1-3]. These receptors play important roles in carcinogenesis and cancer proliferation [4]. Recent data suggest that serum containing insulin glargine (A21Gly, 
B31Arg,B32Arg human insulin) was somewhat more mitogenic to breast cancer cells than serum containing human insulin [5].

The possibility of increased cancer risk in diabetic patients treated with insulin glargine has been addressed in several observational studies [6-10]. In two of these, use of insulin glargine was found to be associated with increased breast cancer risk. However, these studies had methodological limitations leading to several criticisms [11, 12]. The European Medicines Agency (EMA), the US Food and Drug Administration (FDA) and the ADA issued statements stipulating that no conclusions can be drawn from these studies because of their limitations [13-15]. A recent study of 1,340 insulin-treated patients found that high doses of insulin glargine were associated with a fivefold increase in the overall risk of cancer, but the number of participants did not allow sufficient power to investigate the effects on breast cancer risk specifically [10]. Finally, a pooled analysis of data from 31 randomised trials that found no excess cancer risks with insulin glargine was limited by its size and had no exposure beyond 5 years $[16,17]$. Thus, in the absence of new data, the question of whether insulin glargine increases the risk of breast cancer remains unanswered, particularly with longer-term use.

We conducted a large-scale population-based epidemiological study in a cohort of women with type 2 diabetes treated with insulin to assess whether the long-term use of insulin glargine compared with alternative insulin therapy is associated with an elevated risk of breast cancer.

\section{Methods}

Data source This study was conducted using the General Practice Research Database (GPRD), a primary care database from the UK [18]. The GPRD contains the complete primary care medical record for more than 9.9 million people enrolled in more than 545 general practices representative of the UK population. Participating general practitioners have been trained to record medical information including demographic and lifestyle data, medical diagnoses, details of hospital stays, and deaths using a standardised form. Prescriptions written by GPRD physicians are automatically transcribed into the computer record. The recorded information on drug exposures and diagnoses has been validated and proven to be of high quality.

Cohort study design We first formed a base cohort of all female insulin users with type 2 diabetes from all women who had received at least one prescription for any type of insulin between 1 September 2002, the date insulin glargine entered the UK market, and 31 December 2006. Type 2 diabetes was defined as 40 years of age or over, with either a diagnosis or a prescription for an oral hypoglycaemic agent before cohort entry, or no prior use of insulin. All patients had to have at least 1 year of up-to-standard medical history in the GPRD at the time of cohort entry. The insulins included insulin glargine, human long-acting insulins, human biphasic insulins, short-acting analogues and animal insulins. From this cohort, we formed a cohort who had been exposed to insulin glargine and a matched reference insulin-exposed cohort.

First, the glargine-exposed cohort was formed from all women within the base cohort with a prescription for insulin glargine. Cohort entry date was defined as the date of the first insulin glargine prescription in this period, and follow-up was until 31 December 2009. The cohort was divided into first-time insulin users, namely those whose insulin glargine prescription was their first ever insulin prescription, and prevalent insulin users, who had a history of insulin use before cohort entry.

The reference insulin cohort was selected from all nonglargine insulin users of the base cohort, separately for firsttime and prevalent users. The first-time insulin users were simply all included in the reference cohort, and their cohort entry date was defined as the date of the first insulin prescription with follow-up until 31 December 2009. The prevalent users were matched with the glargine-prevalent users on age, calendar time and duration of prior insulin use, according to their birth year strata sizes. Thus, for each glargine-exposed patient, and starting chronologically with the first one, we identified from the non-glargine patients all women of the same birth year stratum $(\leq 1930$, 19311940, 1941-1950 and >1950). Within each birth-matched stratum, we identified women who had an insulin prescription closest to the calendar date of the cohort entry glargine prescription and closest in duration of prior insulin use from the date of this prescription. The cohort entry date for the prevalent members of the reference insulin cohort was then defined as the date of this closest time-matched insulin prescription with follow-up until 31 December 2009.

Women with a diagnosis of gestational diabetes or a history of breast cancer before cohort entry were excluded. Accordingly, all patients in the cohort were observed from cohort entry until the earliest of a first ever diagnosis of breast cancer, death from any cause, end of registration with the general practice, or end of the study period (31 December 2009).

Breast cancer cases We identified all incident cases of breast cancer occurring during follow-up using a validated computerised algorithm developed for a previous study [19]. This algorithm includes PEGASUS and Read codes for breast cancer, as well as combinations of medical procedures, visits or treatments related to this outcome. These consist of mastectomies, lumpectomies, axillary node dissections, consultations with oncologists, chemotherapy 
Fig. 1 Flow chart describing the selection of the cohort of 15,227 women with type 2 diabetes between September 2002 and the end of 2006, followed until 31 December 2009 , all aged 40 years or more, identified from the UK's GPRD

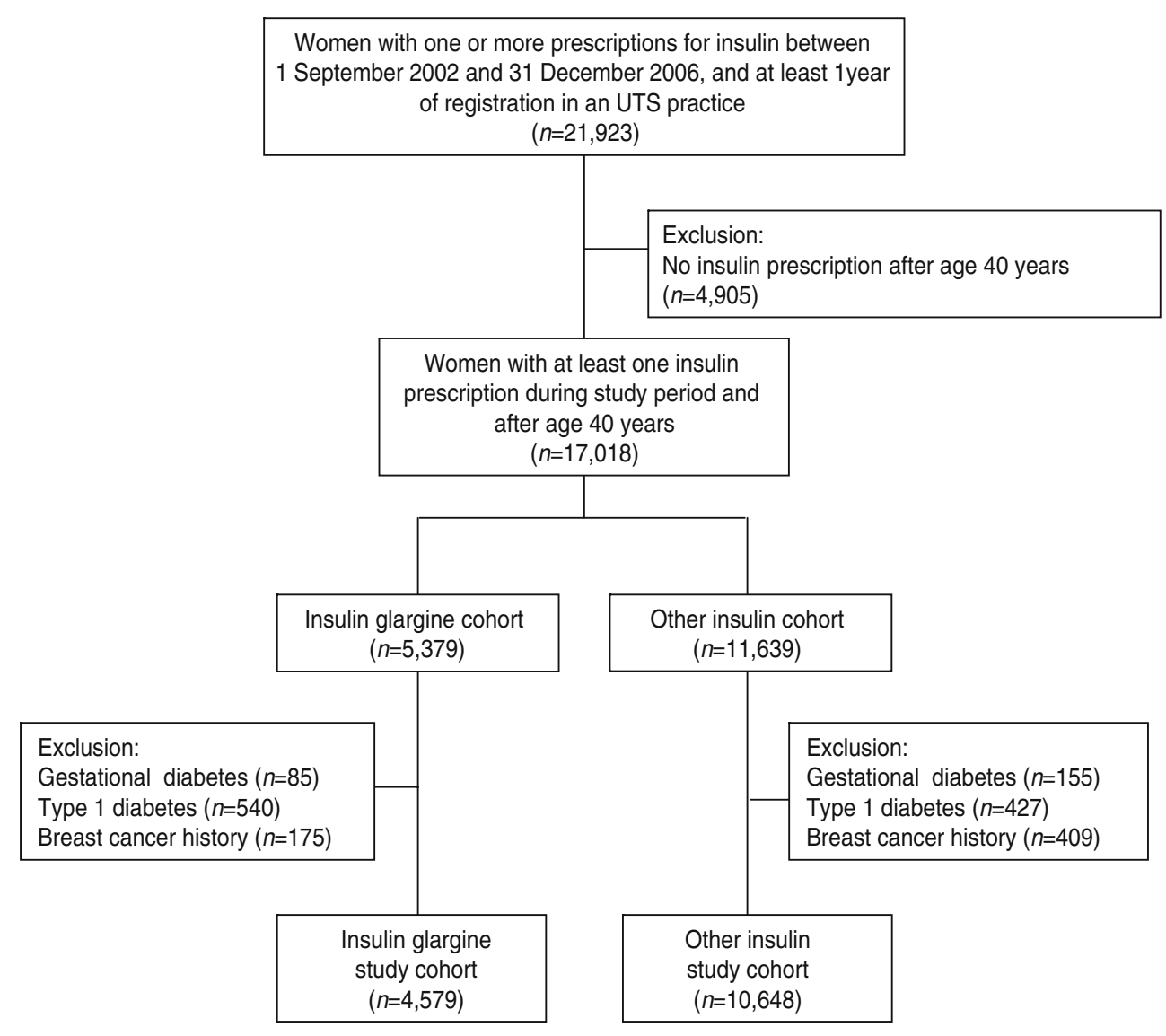

treatments, radiotherapy and use of postoperative antihormone therapy. The validity of this algorithm is high, where over $95 \%$ of diagnoses were confirmed in a review of written records of a random sample of 100 cases [19]. For all identified cases, cohort follow-up stopped on the day of the breast cancer diagnosis.

Covariates The analysis involved adjustment for potential confounding variables known to be associated with breast cancer risk and that may be associated with the choice of insulin therapy. In addition to age at cohort entry, a history of oophorectomy, cancer other than breast or non-melanoma skin cancers, use of hormone replacement therapy (HRT), and smoking any time before cohort entry were considered from the entire medical record including the data collected during the time from registration to cohort entry and the medical history information provided at registration. Durations of diabetes and of insulin use before cohort entry were also determined from the clinical history information recorded by the general practitioner at the time of registration in the practice and from the medical record information before cohort entry. Obesity $\left(B M I \geq 30 \mathrm{~kg} / \mathrm{m}^{2}\right)$ was measured in the 5 years before cohort entry, while excessive alcohol use, $\mathrm{HbA}_{1 \mathrm{c}}$ concentration and use of statins, sulfonylurea, thiazolidinedione and metformin were identified in the year before cohort entry, the latter because of its possible protective effect on the incidence of breast cancer [20].

Statistical analysis The incidence rate of breast cancer over time was estimated by cumulating the person-years of follow-up for the cohort, and the 95\% CIs were based on the Poisson distribution. The Cox proportional hazards model was used to estimate adjusted HRs of breast cancer incidence associated with insulin glargine exposure, along with 95\% CIs, based on an intent-to-treat analysis comparing the insulin glargine-exposed cohort with the reference other-insulin cohort, with time zero taken as the date of cohort entry. All models were adjusted for the potential confounding factors listed above. To control for age, in addition to the age strata used in matching the prevalent cohort, age at cohort entry was also entered in the model as a continuous factor within each age stratum to account for any residual confounding. For the missing data inherently present in the GPRD for BMI, smoking and $\mathrm{HbA}_{1 \mathrm{c}}$, a separate category defining missing information was added in the analysis for each factor. The Cox model was also used to estimate the HR of breast cancer as a function of the time since initiation of insulin glargine. This duration was classified as $<1,1-3,3-5$ and $>5$ years from the respective cohort entry dates for each of the two insulin groups, and a 
Table 1 Baseline characteristics of insulin-treated type 2 diabetic women according to insulin exposure at cohort entry, stratified for first-time vs prevalent insulin use

\begin{tabular}{|c|c|c|c|c|}
\hline \multirow[t]{2}{*}{ Characteristic } & \multicolumn{2}{|c|}{ First-time insulin users } & \multicolumn{2}{|c|}{ Prevalent insulin users } \\
\hline & Glargine & Other insulin & Glargine & Other insulin \\
\hline \multicolumn{5}{|l|}{ Measured at or before cohort entry } \\
\hline Number of patients & 1,604 & 3,086 & 2,975 & 7,562 \\
\hline Age (years) & $66.5 \pm 12.6$ & $64.8 \pm 11.9$ & $60.9 \pm 13.1$ & $61.2 \pm 12.2^{\mathrm{b}}$ \\
\hline Duration of diabetes (years) & $8.3 \pm 6.2$ & $7.7 \pm 7.5$ & $16.9 \pm 12.7$ & $15.3 \pm 10.6$ \\
\hline Duration of prior insulin (years) & - & - & $5.6 \pm 4.6$ & $5.2 \pm 4.0$ \\
\hline \multicolumn{5}{|c|}{ Measured in the 5 years before cohort entry } \\
\hline $\mathrm{BMI} \geq 30 \mathrm{~kg} / \mathrm{m}^{2}(\%)^{\mathrm{a}}$ & 50.8 & 50.1 & 39.5 & 52.3 \\
\hline \multicolumn{5}{|c|}{ Measured in the year before cohort entry } \\
\hline $\mathrm{HbA}_{1 \mathrm{c}}(\%)^{\mathrm{a}}$ & $9.7 \pm 1.9$ & $9.6 \pm 2.1$ & $9.0 \pm 1.7$ & $8.5 \pm 1.8$ \\
\hline $\mathrm{HbA}_{1 \mathrm{c}}(\mathrm{mmol} / \mathrm{mol})^{\mathrm{a}}$ & $82.5 \pm 2.7$ & $81.4 \pm 0.5$ & $74.9 \pm 4.9$ & $69.4 \pm 3.8$ \\
\hline \multicolumn{5}{|l|}{$\mathrm{HbA}_{1 \mathrm{c}}(\% \text { in each category })^{\mathrm{a}}$} \\
\hline$<6.5 \%(<47.5 \mathrm{mmol} / \mathrm{mol})$ & 1.8 & 4.6 & 4.3 & 8.9 \\
\hline $6.5-7.4 \%(47.5-58.0 \mathrm{mmol} / \mathrm{mol})$ & 6.2 & 7.9 & 10.3 & 16.9 \\
\hline $7.5-8.9 \%(58.1-74.8 \mathrm{mmol} / \mathrm{mol})$ & 27.3 & 24.2 & 35.9 & 37.3 \\
\hline$\geq 9 \%(\geq 74.9 \mathrm{mmol} / \mathrm{mol})$ & 64.7 & 63.4 & 49.6 & 36.9 \\
\hline Excessive alcohol use (\%) & 0.44 & 0.94 & 0.50 & 0.25 \\
\hline Metformin use (\%) & 76.5 & 64.1 & 32.3 & 35.9 \\
\hline Sulfonylurea use (\%) & 83.7 & 69.6 & 11.6 & 6.7 \\
\hline Thiazolidinedione use (\%) & 36.2 & 27.9 & 4.3 & 1.5 \\
\hline Statin use $(\%)$ & 65.5 & 58.9 & 56.1 & 58.5 \\
\hline \multicolumn{5}{|c|}{ History of, at any time before index date } \\
\hline Smoking $(\%)^{\mathrm{a}}$ & 49.2 & 51.5 & 46.5 & 44.4 \\
\hline Cancer $(\%)$ & 13.8 & 14.4 & 10.9 & 11.5 \\
\hline Oophorectomy (\%) & 2.5 & 3.5 & 2.9 & 3.0 \\
\hline HRT use (\%) & 25.3 & 25.6 & 29.6 & 19.4 \\
\hline
\end{tabular}

Values are mean \pm SD unless stated otherwise

${ }^{a}$ Among patients with available data for these variables

${ }^{\mathrm{b}}$ Weighted by the inverse of the number of patients matched on age strata

time-dependent Cox model was used to update the risk sets, so that rate ratios are estimated for each of the four duration intervals of glargine insulin, relative to other insulin use in the same time interval. A cubic spline model was also used to produce a smooth curve of the HR as a function of duration [21].

Since the cohort included first-time initiators of insulin and longstanding prevalent users of insulin, the effect of the duration of insulin use after cohort entry may be affected by the duration of insulin use before cohort entry. We thus also analysed the effect of duration separately for prior insulin users to assess this potential source of effect modification. Sensitivity analyses assessed the effect of latency (and possible detection bias) by excluding the first year of follow-up after cohort entry and excluding patients with a history of other cancers before cohort entry. The study was estimated to have over $80 \%$ power to detect rate ratios of 1.5 associated with insulin glargine use. All analyses were conducted using SAS version 9.2 (SAS Institute, Cary, NC, USA).

Ethics approval The study protocol was approved by the Independent Scientific Advisory Committee of the GPRD and by the Jewish General Hospital Research Ethics Committee.

\section{Results}

The base cohort included 15,227 women, 65 years of age on average, after exclusion of 584 with breast cancer before cohort entry (Fig. 1). During the up to 8 years of 
Table 2 Crude and adjusted hazard ratios of breast cancer associated with insulin glargine use relative to other insulin use, by duration of use

\begin{tabular}{|c|c|c|c|c|c|c|}
\hline \multirow{2}{*}{$\begin{array}{l}\text { Duration of insulin } \\
\text { use (years) }\end{array}$} & \multirow{2}{*}{$\begin{array}{l}\text { Breast cancer } \\
\text { cases }\end{array}$} & \multirow[t]{2}{*}{ Person-years } & \multirow{2}{*}{$\begin{array}{l}\text { Rate per } \\
1,000 \text { per year }\end{array}$} & \multicolumn{3}{|c|}{ Hazard ratio $^{a}$} \\
\hline & & & & Crude & Age-adjusted $^{\mathrm{b}}$ & Adjusted $^{\mathrm{c}}(95 \% \mathrm{CI})$ \\
\hline Insulin glargine use & 66 & 18,750 & 3.5 & 0.8 & 1.0 & $1.0(0.7-1.4)$ \\
\hline$<1$ & 18 & 4,332 & 4.2 & 0.7 & 0.9 & $0.9(0.5-1.5)$ \\
\hline $1-3$ & 24 & 7,544 & 3.2 & 0.8 & 1.0 & $1.0(0.6-1.7)$ \\
\hline $3-5$ & 14 & 5,081 & 2.8 & 0.7 & 0.8 & $0.8(0.5-1.6)$ \\
\hline$>5$ & 10 & 1,793 & 5.6 & 1.5 & 1.8 & $1.8(0.8-4.0)$ \\
\hline Other insulin treatment & 180 & 40,741 & 4.4 & 1.0 & 1.0 & Reference \\
\hline$<1$ & 55 & 9,378 & 5.9 & 1.0 & 1.0 & Reference \\
\hline $1-3$ & 62 & 15,709 & 3.9 & 1.0 & 1.0 & Reference \\
\hline $3-5$ & 46 & 11,218 & 4.1 & 1.0 & 1.0 & Reference \\
\hline$>5$ & 17 & 4,436 & 3.8 & 1.0 & 1.0 & Reference \\
\hline
\end{tabular}

${ }^{a}$ Crude (not age-stratified) and adjusted hazard ratios estimated by Cox proportional hazards model

${ }^{\mathrm{b}}$ Age-adjusted according to the age strata used for matching in the cohort of prevalent users

${ }^{\mathrm{c}}$ Cox proportional hazards model adjusted for age categories, age in years, excessive alcohol use, smoking status, obesity $\left(\mathrm{BMI} \geq 30 \mathrm{~kg} / \mathrm{m}^{2}\right)$, $\mathrm{HbA}_{1 \mathrm{c}}$, diabetes duration, duration of insulin use, oophorectomy, history of cancer, use of HRT, sulfonylureas, thiazolidinediones, metformin and statins, all before cohort entry

follow-up, 246 developed breast cancer, for an overall incidence rate of breast cancer of 4.1 (95\% CI 3.6-4.7) per 1,000 per year.

The glargine-exposed cohort included 4,579 women and the age-matched other-insulin cohort included 10,648 women, shown in Table 1 separately for firsttime and prevalent insulin users. The first-time insulin glargine users were older, but had had diabetes for a shorter time than their prevalent counterparts. While the first-time glargine insulin users were generally similar to the first-time users of other insulins, the prevalent glargine users were leaner, had less well controlled diabetes as indicated by their $\mathrm{HbA}_{1 \mathrm{c}}$ level, and used more sulfonylureas, thiazolidinediones and HRT, compared with the age-matched prevalent users of other insulins (Table 1).

Table 3 Crude and adjusted hazard ratios of breast cancer associated with duration of insulin glargine use relative to other insulin use, among first-time users of insulin at cohort entry

\begin{tabular}{|c|c|c|c|c|c|c|}
\hline \multirow{2}{*}{$\begin{array}{l}\text { Duration of insulin } \\
\text { use (years) }\end{array}$} & \multirow{2}{*}{$\begin{array}{l}\text { Breast cancer } \\
\text { cases }\end{array}$} & \multirow[t]{2}{*}{ Person-years } & \multirow{2}{*}{$\begin{array}{l}\text { Rate per } \\
1,000 \text { per year }\end{array}$} & \multicolumn{3}{|c|}{ Hazard ratio $^{a}$} \\
\hline & & & & Crude & Age-adjusted $^{\mathrm{b}}$ & Adjusted $^{\mathrm{c}}(95 \% \mathrm{CI})$ \\
\hline Insulin glargine use & 18 & 6,094 & 3.0 & 0.6 & 0.7 & $0.8(0.3-2.1)$ \\
\hline$<1$ & 6 & 1,513 & 4.0 & 0.7 & 0.8 & $1.0(0.3-3.1)$ \\
\hline $1-3$ & 8 & 2,601 & 3.1 & 0.7 & 0.7 & $0.9(0.3-2.7)$ \\
\hline $3-5$ & 4 & 1,587 & 2.5 & 0.6 & 0.6 & $0.8(0.2-3.1)$ \\
\hline$>5$ & 0 & 393 & 0.0 & 0.0 & 0.0 & Not estimable \\
\hline Other insulin treatment & 60 & 12,262 & 4.9 & 1.0 & 1.0 & Reference \\
\hline$<1$ & 16 & 2,886 & 5.5 & 1.0 & 1.0 & Reference \\
\hline $1-3$ & 23 & 4,940 & 4.7 & 1.0 & 1.0 & Reference \\
\hline $3-5$ & 14 & 3,267 & 4.3 & 1.0 & 1.0 & Reference \\
\hline$>5$ & 7 & 1,169 & 6.0 & 1.0 & 1.0 & Reference \\
\hline
\end{tabular}

${ }^{\text {a }}$ Crude (not age-stratified) and adjusted hazard ratios estimated by Cox proportional hazards model

${ }^{\mathrm{b}}$ According to the age strata used for matching in the cohort of prevalent users

${ }^{\mathrm{c}}$ Cox proportional hazards model adjusted for age categories, age in years, excessive alcohol use, smoking status, obesity $\left(\mathrm{BMI} \geq 30 \mathrm{~kg} / \mathrm{m}^{2}\right)$, $\mathrm{HbA}_{1 \mathrm{c}}$, diabetes duration, oophorectomy, history of cancer, use of HRT, sulfonylureas, thiazolidinediones, metformin and statins, all before cohort entry 
Table 4 Crude and adjusted hazard ratios of breast cancer associated with duration of insulin glargine use relative to other insulin use, among previous users of insulin at cohort entry

\begin{tabular}{|c|c|c|c|c|c|c|}
\hline \multirow{2}{*}{$\begin{array}{l}\text { Duration of insulin } \\
\text { use (years) }\end{array}$} & \multirow{2}{*}{$\begin{array}{l}\text { Breast cancer } \\
\text { cases }\end{array}$} & \multirow[t]{2}{*}{ Person-years } & \multirow{2}{*}{$\begin{array}{l}\text { Rate per } \\
1,000 \text { per year }\end{array}$} & \multicolumn{3}{|c|}{ Hazard ratio $^{a}$} \\
\hline & & & & Crude & Age-adjusted $^{\mathrm{b}}$ & Adjusted $^{\mathrm{c}}(95 \% \mathrm{CI})$ \\
\hline Insulin glargine use & 48 & 12,656 & 3.8 & 0.9 & 1.1 & $1.0(0.7-1.5)$ \\
\hline$<1$ & 12 & 2,819 & 4.3 & 0.7 & 0.8 & $0.8(0.4-1.6)$ \\
\hline $1-3$ & 16 & 4,942 & 3.2 & 0.9 & 1.0 & $1.0(0.6-1.8)$ \\
\hline $3-5$ & 10 & 3,495 & 2.9 & 0.7 & 0.8 & $0.8(0.4-1.6)$ \\
\hline$>5$ & 10 & 1,400 & 7.1 & 2.3 & 2.7 & $2.7(1.1-6.5)$ \\
\hline Other insulin treatment & 120 & 28,478 & 4.2 & 1.0 & 1.0 & Reference \\
\hline$<1$ & 39 & 6,491 & 6.0 & 1.0 & 1.0 & Reference \\
\hline $1-3$ & 39 & 10,770 & 3.6 & 1.0 & 1.0 & Reference \\
\hline $3-5$ & 32 & 7,951 & 4.0 & 1.0 & 1.0 & Reference \\
\hline$>5$ & 10 & 3,267 & 3.1 & 1.0 & 1.0 & Reference \\
\hline
\end{tabular}

${ }^{\text {a }}$ Crude (not age-stratified) and adjusted hazard ratios estimated by Cox proportional hazards model

${ }^{\mathrm{b}}$ According to the age strata used for matching in the cohort of prevalent users

${ }^{\mathrm{c}}$ Cox proportional hazards model adjusted for age categories, age in years, excessive alcohol use, smoking status, obesity (BMI $\left.\geq 30 \mathrm{~kg} / \mathrm{m}^{2}\right)$, $\mathrm{HbA}_{1 \mathrm{c}}$, diabetes duration, duration of insulin use, oophorectomy, history of cancer, use of HRT, sulfonylureas, thiazolidinediones, metformin and statins, all before cohort entry

Women in the glargine-exposed cohort received on average 5.8 prescriptions of insulin glargine per year during follow-up and 3.6 prescriptions of short-acting analogues, as well as biphasic insulin ( 0.9 per year). The reference group received primarily human biphasic insulin (3.6 prescriptions per year) and analogue biphasic (2.7 per year), as well as human long-acting (1.3 per year) and short-acting ( 0.9 per year) analogues. The dosing information for most of the prescriptions entered in the medical

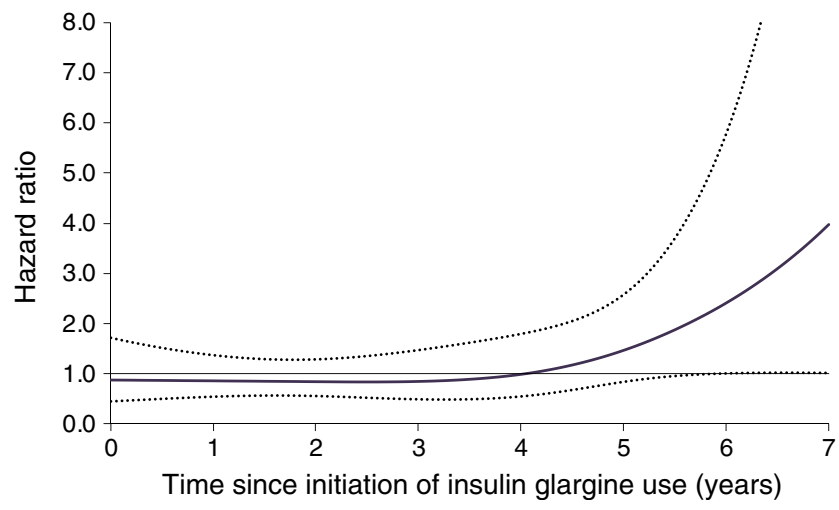

Fig. 2 Smooth cubic spline curve of the adjusted hazard ratio of breast cancer incidence (solid line) and 95\% confidence limits (dotted lines) as a function of the time since initiation of insulin glargine use relative to the same duration for other insulin use, among previous users of insulin at cohort entry record was 'as directed', thus not permitting any doseresponse analyses.

After adjustment for differences in the covariates, use of insulin glargine was not associated with an increased risk of breast cancer (HR 1.0; 95\% CI 0.7-1.4), relative to other insulins (Table 2). The risk was not increased in the first 5 years of use (overall HR $0.9 ; 95 \%$ CI $0.7-1.3$ ), but there is a trend towards an elevated risk after 5 years or more of glargine use (HR 1.8; 95\% CI 0.8-4.0).

Stratification by the use of insulin before cohort entry shows in Table 3 that, among first-time insulin users, the risk of breast cancer is not elevated with insulin glargine use relative to other insulins (HR $0.8 ; 95 \%$ CI $0.3-2.1$ ). In particular, no cases of breast cancer were observed 5 or more years after glargine insulin initiation, although this stratum only had 393 person-years of follow-up.

Table 4 shows that, among previous insulin users, the risk of breast cancer is not elevated in the first 5 years of insulin glargine use (overall HR 0.9; 95\% CI 0.6-1.3). However, in these previous users who had already been receiving insulin for an average of 5.6 years before starting glargine, the risk increases significantly after 5 years of insulin glargine use (HR 2.7; 95\% CI 1.1-6.5). Figure 2 displays the smooth cubic spline curve of the hazard ratio as a function of duration of glargine use relative to other insulins, among previous insulin users. The lower 95\% confidence band of this risk curve is only on the borderline of statistical significance after 5 years of glargine use, 
although this effect on the curve may have resulted from the generally lower risk during the early years of use.

The sensitivity analyses of this estimate of the risk after 5 years of insulin glargine use in previous insulin users produced similar results after exclusion of exposures in the first year of follow-up (HR 2.5; 95\% CI 1.0-6.1), but the risk increase was somewhat numerically higher after exclusion of patients with a history of other cancers before cohort entry (HR 3.9; 95\% CI 1.5-10.5).

\section{Discussion}

Using a large cohort of women with insulin-treated type 2 diabetes, observed for up to 8 years, we found that, in the short term, the use of insulin glargine was not associated with an increased risk of breast cancer. However, after more than 5 years, the risk tended to be increased in the longstanding users of insulin before initiation of insulin glargine, but not in women whose first insulin was glargine.

Some experimental studies have suggested that insulin stimulation of growth of cancers is mediated by insulin stimulation of IGF-I receptors [22]. While this possibility is not excluded by recent data, the documentation of expression of insulin receptors by malignant cells indicates that even insulins that have no IGF-I receptor agonist activity could stimulate proliferation [23, 24]. At least two non-mutually exclusive mechanisms by which endogenous or administered insulin may contribute to cancer risk have been proposed. One mechanism, which may result in a relatively short-term effect, does not involve an effect of insulin on carcinogenesis, but rather a stimulatory effect of insulin on the growth rate of the subset of cancers that are present but not yet of a size that can be diagnosed. This mechanism has features in common with the effects of postmenopausal HRT or pregnancy on breast cancer risk, in that effects may be evident in under 2 years [25], and is worthy of consideration given the high frequency of subclinical cancers in middle-aged women [26]. A second mechanism involves an effect on the process of stepwise carcinogenesis where the relevant receptors are on breast epithelial cells at risk of transformation. Stepwise carcinogenesis is a slow process involving gradual accumulation of genetic damage leading to transformation. Insulin exposure is hypothesised to facilitate stepwise carcinogenesis not by any mutagenic action, but rather because activation of insulin or IGF-I receptors (or hybrid receptors) can increase the probability of survival of partially transformed cells [27]. This mechanism is compatible with an influence of longterm exposure on risk, as observed in the present study.

If insulin exposure increases breast cancer risk, is it plausible that synthetic insulins in general, or glargine in particular, could have a greater effect than endogenous insulin or other administered insulins? There is laboratory evidence that various insulins differ from each other in receptor-binding characteristics and mitogenic effects [2, $28,29]$. An early study using the insulin analogue B10ASP showed greater carcinogenic action of this engineered insulin than human insulin $[30,31]$. These data led to the decision not to develop B10ASP for clinical use. This experience supports the plausibility of the hypothesis that exposure to different insulins could have different effects on cancer-related outcomes, but there is a paucity of in vivo experimental data to rigorously examine effects of currently used insulin analogues on cancer behaviour. A recent study suggests that serum from patients receiving insulin glargine is somewhat more mitogenic for breast cancer cells in vitro than serum from patients receiving other insulins [5]. However, many investigators currently view the hypothesis that glargine is more mitogenic than other insulins in vivo to be plausible, but unproven. In addition to differences between insulins in receptor-binding characteristics, we cannot exclude the possibility that pharmacokinetics play a role; physiological insulin secretion is pulsatile, and there are many hours per day where exposure of tissues to insulin is minimal. In contrast, long-acting insulin use provides a more continuous exposure, and it is conceivable that this is relevant for oncological end points. In this case, it is plausible that all long-acting insulins, rather than glargine in particular, would be associated with increased cancer risk. Therefore additional studies of other insulins are justified, but available data are limited. For example, data concerning insulin detemir (B29Lys(E-tetradecanoyl), desB30 human insulin), another long-acting basal insulin analogue, were insufficient to allow a conclusion on cancer safety [32]. Similarly, the pooled analysis of data from randomised trials of insulin glargine that found no excess cancer risks was also deemed insufficient to conclude safety $[16,17]$. Observational studies of these long-term effects with newer insulins will thus be necessary but will take some time to conduct.

A possible methodological explanation for the increased risk of breast cancer among longstanding users of glargine is an effect of the longer overall duration of insulin use, as women initiating glargine had already been on insulin for an average of 5.6 years. This is unlikely, however, since the comparison group of users of other insulins had also been on insulin for 5.2 years before cohort entry, making it unlikely that the increase in risk is due to a general insulin duration effect. Another possible explanation is an imbalance in baseline characteristics, as the glargine users appear to be different from the other insulin users in terms of factors including control of glycaemia and HRT use. It is, however, unlikely, after adjustment, that residual confounding alone would explain the almost threefold increase in risk. Indeed, first, diabetes severity and duration are only associated with a 
minor or even no increase in breast cancer risk [33]. Second, if a characteristic of the high-risk group other than glargine use was critical in conferring such an increased risk, the increase in risk would have been observed immediately in the first year of glargine exposure, which was not the case even for the first 5 years. In fact, the somewhat lower risk in the first year is not only indicative of the absence of such residual or unmeasured confounding, but suggests instead an even lower baseline risk for the insulin glargine users.

Our study addressed some limitations of previous studies $[11,12]$. First, we focused specifically on type 2 diabetes and evaluated breast cancer only, the subject of contradictory findings [6-9]. Second, the previous observational studies were limited by their short follow-up, of at most 5 years [79]. Moreover, the pooled analysis of data from 31 randomised trials of insulin glargine was driven by a single large trial with at most 5 years of exposure [16]. In our study, the women were followed for up to 8 years, which permitted the analysis of the risk beyond 5 years after glargine initiation.

Our observational study has strengths and limitations. First, the study is based on a large cohort of over 15,000 female insulin users with type 2 diabetes observed over an 8-year span. It provided sufficient power to detect the excess risks under study, with 66 cases of breast cancer exposed to glargine insulin, larger than the number among patients with type 2 diabetes in the previous studies. However, stratification by the use of insulin before cohort entry provided an insufficient number of women whose first ever insulin was glargine. Consequently, we observed no cases of breast cancer 5 or more years after glargine insulin initiation out of only 393 personyears of follow-up, compatible with an incidence rate as high as 7.6 per 1,000 per year from the upper limit of the $95 \%$ confidence interval. Second, selection bias was avoided in two ways: by the completeness from the population-based cohort composed of all women with type 2 diabetes treated with insulin in the GPRD, which also provides generalisability, and by matching on cohort entry time. Moreover, stratification according to first-time and prevalent use of insulin was important to account for the history of insulin use. Because the GPRD uses pre-recorded exposure histories, the possibility of recall bias in terms of exposure to the different insulin therapies is avoided. We were able to adjust for the effects of BMI, excessive alcohol use and smoking, measures often absent from other databases. We used an intent-to-treat exposure definition which by definition does not consider treatment discontinuations that may have occurred during follow-up. This approach, however, avoids biases related to reverse causation, where glargine may have been stopped at early signs or symptoms of breast cancer, so that treatment termination would not be independent of the outcome. Thus the threat to internal validity caused by reverse causation may be far greater than that of using the intent-to-treat approach, which typically has the effect over the longer term of diluting the estimates towards the null, which is not an issue in our study. Moreover, while compliance with the treatment regimen is unknown as exposure comes from prescriptions, it is unlikely that there was much misclassification, as insulin treatment is more compliance-prone. It was not possible to accurately evaluate the effect of dose, as dose information was not entered in the GPRD files for the majority of patients. Our study did not have data on certain breast cancer risk factors, such as diet, physical activity and race/ethnicity. Finally, the role of chance cannot be dismissed in the finding of the elevated HR of 2.7 with a $p$ value of 0.031 , since this $p$ value is associated with a stratified analysis that repeated this analysis twice for those with and without a history of insulin use before cohort entry.

In conclusion, while use of insulin glargine does not appear to increase the risk of breast cancer among women with type 2 diabetes in the short term, our data suggest that its longer term use may do so. Prospective studies of this issue would be more definitive, but are probably impractical to undertake. As there is some controversy in claiming that long-acting analogues are superior to conventional insulins in reducing long-term diabetes morbidity and mortality $[34,35]$, the available evidence concerning cancer risk among long-term glargine users must be weighed against advantages glargine may have over other insulins in terms of patient preferences and hypoglycaemia risk $[34,35]$.

Acknowledgements This research was funded by grants from the Canadian Institutes of Health Research (CIHR) and the Canadian Foundation for Innovation (CFI). S. Suissa and M. Pollak participated in advisory committee meetings organised by Sanofi-Aventis, a maker of insulin glargine, and M. Pollak has consulted for Novo Nordisk. S. Suissa is the recipient of a James McGill Chair, M. Pollak holds the Alexander Goldfarb Chair in Oncology and L. Azoulay is the recipient of a Chercheur-boursier award from the Fonds de la recherche en santé du Quebec (FRSQ). S. Suissa, principal investigator independent of any commercial funder, had full access to all of the data in the study and takes responsibility for the integrity of the data and the accuracy of the data analysis.

Duality of interest The authors declare that there is no duality of interest associated with this manuscript.

\section{References}

1. Werner H, Weinstein D, Bentov I (2008) Similarities and differences between insulin and IGF-I: structures, receptors, and signalling pathways. Arch Physiol Biochem 114:17-22

2. Kurtzhals P, Schaffer L, Sorensen A et al (2000) Correlations of receptor binding and metabolic and mitogenic potencies of insulin analogs designed for clinical use. Diabetes 49:999-1005

3. Sommerfeld MR, Muller G, Tschank G et al (2010) In vitro metabolic and mitogenic signaling of insulin glargine and its metabolites. PLoS ONE 5:e9540

4. Baserga R (1999) The IGF-I receptor in cancer research. Exp Cell Res 253:1-6

5. Mayer D, Chantelau E (2010) Treatment with insulin glargine (Lantus) increases the proliferative potency of the serum of 
patients with type-1 diabetes: a pilot study on MCF-7 breast cancer cells. Arch Physiol Biochem 116:73-78

6. Hemkens LG, Grouven U, Bender R et al (2009) Risk of malignancies in patients with diabetes treated with human insulin or insulin analogues: a cohort study. Diabetologia 52:1732-1744

7. Colhoun HM (2009) Use of insulin glargine and cancer incidence in Scotland: a study from the Scottish Diabetes Research Network Epidemiology Group. Diabetologia 52:1755-1765

8. Jonasson JM, Ljung R, Talback M, Haglund B, Gudbjornsdottir S, Steineck G (2009) Insulin glargine use and short-term incidence of malignancies: a population-based follow-up study in Sweden. Diabetologia 52:1745-1754

9. Currie CJ, Poole CD, Gale EA (2009) The influence of glucoselowering therapies on cancer risk in type 2 diabetes. Diabetologia 52:1766-1777

10. Mannucci E, Monami M, Balzi D et al (2010) Doses of insulin and its analogues and cancer occurrence in insulin-treated type 2 diabetic patients. Diabetes Care 33:1997-2003

11. Pocock SJ, Smeeth L (2009) Insulin glargine and malignancy: an unwarranted alarm. Lancet 374:511-513

12. Hernandez-Diaz S, Adami HO (2010) Diabetes therapy and cancer risk: causal effects and other plausible explanations. Diabetologia 53:802-808

13. US Department of Health \& Human Services. Early communication about safety of Lantus (insulin glargine). MedWatch The FDA Safety Information and Adverse Event Reporting Program (July 1, 2009). www.fda.gov/Drugs/DrugSafety/PostmarketDrugSafetyInforma tionforPatientsandProviders/DrugSafetyInformationforHeathcare Professionals/ucm169722.htm. Accessed 3 May 2011.

14. European Medicines Agency update on safety of insulin glargine. European Medicines Agency (July 23, 2009). www.ema.europa. eu/docs/en_GB/document_library/Press_release/2009/11/ WC500010016.pdf. Accessed 3 May 2011.

15. American Diabetes Association. Statement from the American Diabetes Association Related to Studies Published in 'Diabetologia'. NeLM news service (June 26, 2009). http://www.diabetes. org/for-media/2009/statement-from-the-american-2009-1.html. Accessed 3 May 2011.

16. Home PD, Lagarenne P (2009) Combined randomised controlled trial experience of malignancies in studies using insulin glargine. Diabetologia 52:2499-2506

17. Hemkens LG, Grouven U, Bender R, Sawicki PT (2010) Insufficient evaluation of adverse events is not a proof of safety. Diabetologia 53:790-792

18. Walley T, Mantgani A (1997) The UK General Practice Research Database. Lancet 350:1097-1099

19. Opatrny L, Dell'Aniello S, Assouline S, Suissa S (2008) Hormone replacement therapy use and variations in the risk of breast cancer. BJOG 115:169-175

20. Bodmer M, Meier C, Krahenbuhl S, Jick SS, Meier CR (2010) Long-term metformin use is associated with decreased risk of breast cancer. Diabetes Care 33:1304-1308
21. Heinzl H, Kaider A, Zlabinger G (1996) Assessing interactions of binary time-dependent covariates with time in cox proportional hazards regression models using cubic spline functions. Stat Med 15:2589-2601

22. Yehezkel E, Weinstein D, Simon M, Sarfstein R, Laron Z, Werner H (2010) Long-acting insulin analogues elicit atypical signalling events mediated by the insulin receptor and insulin-like growth factor-I receptor. Diabetologia 53:26672675

23. Law JH, Habibi G, Hu K et al (2008) Phosphorylated insulin-like growth factor-i/insulin receptor is present in all breast cancer subtypes and is related to poor survival. Cancer Res 68:1023810246

24. Cox ME, Gleave ME, Zakikhani M et al (2009) Insulin receptor expression by human prostate cancers. Prostate 69:33-40

25. Lambe M, Hsieh C, Trichopoulos D, Ekbom A, Pavia M, Adami HO (1994) Transient increase in the risk of breast cancer after giving birth. N Engl J Med 331:5-9

26. Welch HG, Black WC (1997) Using autopsy series to estimate the disease "reservoir" for ductal carcinoma in situ of the breast: how much more breast cancer can we find? Ann Intern Med 127:10231028

27. Pollak M (2008) Insulin and insulin-like growth factor signalling in neoplasia. Nat Rev Cancer 8:915-928

28. Hansen BF, Danielsen GM, Drejer K et al (1996) Sustained signalling from the insulin receptor after stimulation with insulin analogues exhibiting increased mitogenic potency. Biochem J 315:271-279

29. Shukla A, Grisouard J, Ehemann V, Hermani A, Enzmann H, Mayer D (2009) Analysis of signaling pathways related to cell proliferation stimulated by insulin analogs in human mammary epithelial cell lines. Endocr Relat Cancer 16:429-441

30. Jorgensen L, Dideriksen L, Drejen K (1992) Carcinogenic effect of human insulin and B10Asp in female rats. Diabetologia 35 (Suppl 1):A3

31. Brange J, Ribel U, Hansen JF et al (1988) Monomeric insulins obtained by protein engineering and their medical implications. Nature 333:679-682

32. Dejgaard A, Lynggaard H, Rastam J, Krogsgaard TM (2009) No evidence of increased risk of malignancies in patients with diabetes treated with insulin detemir: a meta-analysis. Diabetologia 52:2507-2512

33. Michels KB, Solomon CG, Hu FB et al (2003) Type 2 diabetes and subsequent incidence of breast cancer in the Nurses' Health Study. Diabetes Care 26:1752-1758

34. Singh SR, Ahmad F, Lal A, Yu C, Bai Z, Bennett H (2009) Efficacy and safety of insulin analogues for the management of diabetes mellitus: a meta-analysis. CMAJ 180:385-397

35. Horvath $\mathrm{K}$, Jeitler $\mathrm{K}$, Berghold $\mathrm{A}$ et al. Long-acting insulin analogues vs NPH insulin (human isophane insulin) for type 2 diabetes mellitus. Cochrane Database Syst Rev 2007, Issue 2. Art. No.: CD005613. doi:10.1002/14651858.CD005613.pub3 